\title{
Effect of laser phototherapy in the prevention and treatment of chemo- induced mucositis in hamsters
}

Talita Christine Camilo Lopez(a) Manoela Domingues Martins ${ }^{(b)}$ Vanessa Christina Santos Pavesi(c) Leila Soares Ferreira ${ }^{(d)}$ Sandra Kalil Bussadori(c) Maria Stella Moreira ${ }^{(d)}$ Márcia Martins Marques ${ }^{(a)}$

(a) Department of Restorative Dentistry, School of Dentistry, Univ de São Paulo - USP, São Paulo, SP, Brazil.

(b) Department of Oral Pathology, School of Dentistry, Univ Federal do Rio Grande do Sul - UFRGS, Porto Alegre, RS, Brazil.

(c) Postgraduate Program in Rehabilitation Sciences, School of Dentistry, Univ Nove de Julho - Uninove, São Paulo, SP, Brazil.

(d) Postgraduate Program in Dentistry, School of Dentistry, Univ Ibirapuera - Unib, São Paulo, SP, Brazil.
Declaration of Interests: The authors certify that they have no commercial or associative interest that represents a conflict of interest in connection with the manuscript.

Corresponding Author:

Márcia Martins Marques

E-mail:mmmarques@usp.br

Submitted: Nov 29, 2012

Accepted for publication: Mar 13, 2013

Last revision: Apr 12, 2013
Abstract: The aim of this study was to investigate the effect of laser phototherapy (LPT) in the prevention and/or treatment of oral mucositis induced by 5-fluorouracil (5-FU; Eurofarma, São Paulo, Brazil) in hamsters. Ninety-six hamsters were divided into four groups $(n=24)$ : Control (no treatment); Preventive [LPT from day (D) D-5 to D+5]; Therapeutic (LPT from $\mathrm{D}+5$ to $\mathrm{D}+15$ ); and Combined (preventive plus therapeutic LPT from D-5 to D+15). The animals received an intraperitoneal injection of 5-FU on Days 0 and 2. The pouch mucosa was scratched on Days 3 and 4. The irradiation parameters were: indium-gallium-aluminum-phosphide (InGaAlP) diode laser (MM Optics, São Carlos, Brazil) $(660 \mathrm{~nm})$, beam area of $0.036 \mathrm{~cm}^{2}, 40 \mathrm{~mW}, 1.11 \mathrm{~W} / \mathrm{cm}^{2}, 6.6 \mathrm{~J} / \mathrm{cm}^{2}$, power density applied daily of $39.6 \mathrm{~J} / \mathrm{cm}^{2}$, in punctual mode (six points and six seconds per point) and contact mode, one application per day. The animals were sacrificed on Days 0, 5, 10 and $15(\mathrm{n}=6)$ and weighed, and the pouch mucosa was removed for histopathological analysis. Clinical and corresponding histological scores were compared using ANOVA and Tukey's test $(\mathrm{p} \leq 0.05)$. Similar weight losses ranging from $5 \%$ to $10 \%$ occurred in all groups. The therapeutic group had significantly lower clinical and histological scores than the other groups at Day 10. This study showed that positive effects on oral mucositis management were obtained only when LPT was applied in the therapeutic protocol (from $\mathrm{D}+5$ to $\mathrm{D}+15$ after chemotherapy).

Descriptors: Stomatitis; Lasers; Therapeutics; Radiotherapy.

\section{Introduction}

Oral mucositis is a complication resulting from radiotherapy and/or chemotherapy for cancer treatment. ${ }^{1}$ Clinically, mucositis is graded in stages ranging from minimal symptoms of erythematous lesions to severe ulceration and pain, which may require the cancer treatment to be discontinued, thereby leading to a reduction in quality of life and/or patient

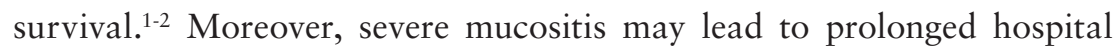
stays and requires special care, such as the intravenous infusion of barbiturates or other drugs and parenteral nutrition, leading to a significant increase in hospital costs. ${ }^{2}$

The treatment is generally palliative, aimed at curbing symptoms and controlling infection and bleeding. The most common types of treatment for oral mucositis are topical antimicrobial agents, cytokines to stimulate 
the medulla, vitamins, growth factors, corticoid and non-alcoholic mouth washes, supplementary amino acids, cryotherapy, and treatment with laser phototherapy (LPT). ${ }^{3-6}$

Studies on the effects of LPT, either alone or in combination with other therapies, conducted in patients with immunosuppression, have shown that LPT promotes a reduction in pain intensity and mucositis severity, acceleration of wound healing, analgesic effects and an increase in the salivary flow rate, with no side effects. . $^{3-4,7-8}$

Studies involving animal models allow a better understanding of the pathobiology of mucositis and a more in-depth evaluation of the effects of therapies for the prevention and treatment of this disease. The model for induced mucositis in hamsters was initially proposed by Sonis et al. ${ }^{9}$ and has proven useful in preclinical studies on new treatment options for mucositis. ${ }^{9-15}$ This model was applied in a few studies for analyzing the effects of LPT in the prevention $^{13}$ or therapeutics ${ }^{13-15}$ of oral mucositis, with positive findings. Although these studies have obtained promising outcomes, the effects of combined protocols (e.g. preventive plus therapeutic) have not been studied thus far. Thus, the aim of the present study was to analyze the effects of three LPT protocols in preventing and treating oral mucositis induced by 5 -fluorouracil (5-FU) in hamsters, including a combined protocol.

\section{Methodology}

The methodology employed in the present study received the approval of the Ethics Committee of the Universidade Nove de Julho (São Paulo, Brazil) under protocol \#AN005/2009. Ninety-six male golden Syrian hamsters with a mean body mass of $150 \mathrm{~g}$ and an age of 8 weeks were used. They were fed a standard laboratory diet and water ad libitum.

\section{Experimental groups}

The hamsters were randomly divided into four groups:

- G1 - Control (no treatment);

- G2 - Preventive [submitted to LPT from D-5 to $\mathrm{D}+5$ ( 5 days before to 5 days after 5 -FU infusion, 5-fluorouracil, Eurofarma, São Paulo, Brazil)];
- G3 - Therapeutic [submitted to LPT from D+5 to $\mathrm{D}+15$ (5 to 15 days after 5-FU infusion)];

- G4 - Combined [subjected to LPT from D-5 to $\mathrm{D}+15$ (preventive + therapeutic)].

\section{Mucositis induction protocol}

Before induction of mucositis, the animals were sedated with isoflurane gas. For the induction of mucositis, $60 \mathrm{mg} / \mathrm{kg}$ of the chemotherapy drug 5 -FU was administered to each animal intraperitoneally on Day 0 , and $40 \mathrm{mg} / \mathrm{kg}$ was administered on Day 2 (protocol proposed by Sonis et al. ${ }^{9}$ and modified by Leitão et $a l .^{12}$ ). The right pouch mucosa was scratched by the same operator on Days 3 and 4 using the tip of a sterile needle twice in a linear movement across the everted cheek pouch. Based on this protocol, oral mucositis establishment occurs one day after the second scratch session (i.e. $\mathrm{D}+5$ ). ${ }^{9,12}$

\section{Laser phototherapy protocol}

The animals receiving laser therapy were sedated with isoflurane gas. LPT was performed with a continuous indium-gallium-aluminum-phosphide (InGaAlP) diode laser $(\lambda=660 \mathrm{~nm}$, and beam spot size of $0.036 \mathrm{~cm}^{2}$; MM Optics, São Carlos, Brazil). The irradiation was punctual in contact mode, perpendicular to the right pouch mucosa in six points per pouch using the following parameters: $40 \mathrm{~mW}$, $1.11 \mathrm{~W} / \mathrm{cm}^{2}, 6.6 \mathrm{~J} / \mathrm{cm}^{2}$; power density of $39.6 \mathrm{~J} / \mathrm{cm}^{2}$ applied daily, 6 seconds per point and $0.24 \mathrm{~J}$ per point.

One LPT application was performed daily starting on different days according to the experimental groups. The range of LPT applications was based on the Sonis et al. ${ }^{9}$ protocol that describes the $\mathrm{D}+5$ as the initial time of oral mucositis establishment (Figure 1).

\section{Observations and determinations}

The animals were weighed at the beginning and end of the experiment and monitored daily to determine morbidity and mortality. On Days $0,5,10$ and 15 , the pouch mucosa of six animals per group was everted and photographed for subsequent clinical analysis. The animals were sacrificed in $\mathrm{a} \mathrm{CO}_{2}$ chamber. The photographs of the right pouch muco- 
Figure 1 - Illustrative photographs showing the irradiation procedure:

(A) six points where the pouch mucosa was irradiated; (B) positioning of laser equipment tip.
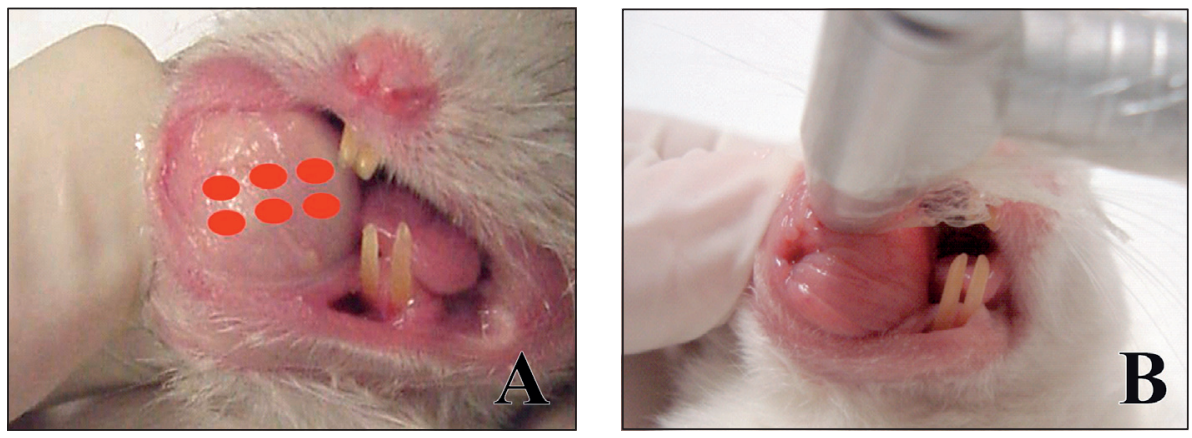

Figure 2 - Clinical aspects relevant to the different clinical scores from Score 0 to Score 3: Score 0 - No alterations are observed; Score 1 - Moderate erythema and hyperemia; Score 2 - Severe erythema and hyperemia, with hemorrhagic areas, and small ulcers; Score 3 - The pouch mucosa exhibits extensive ulceration and abscess.
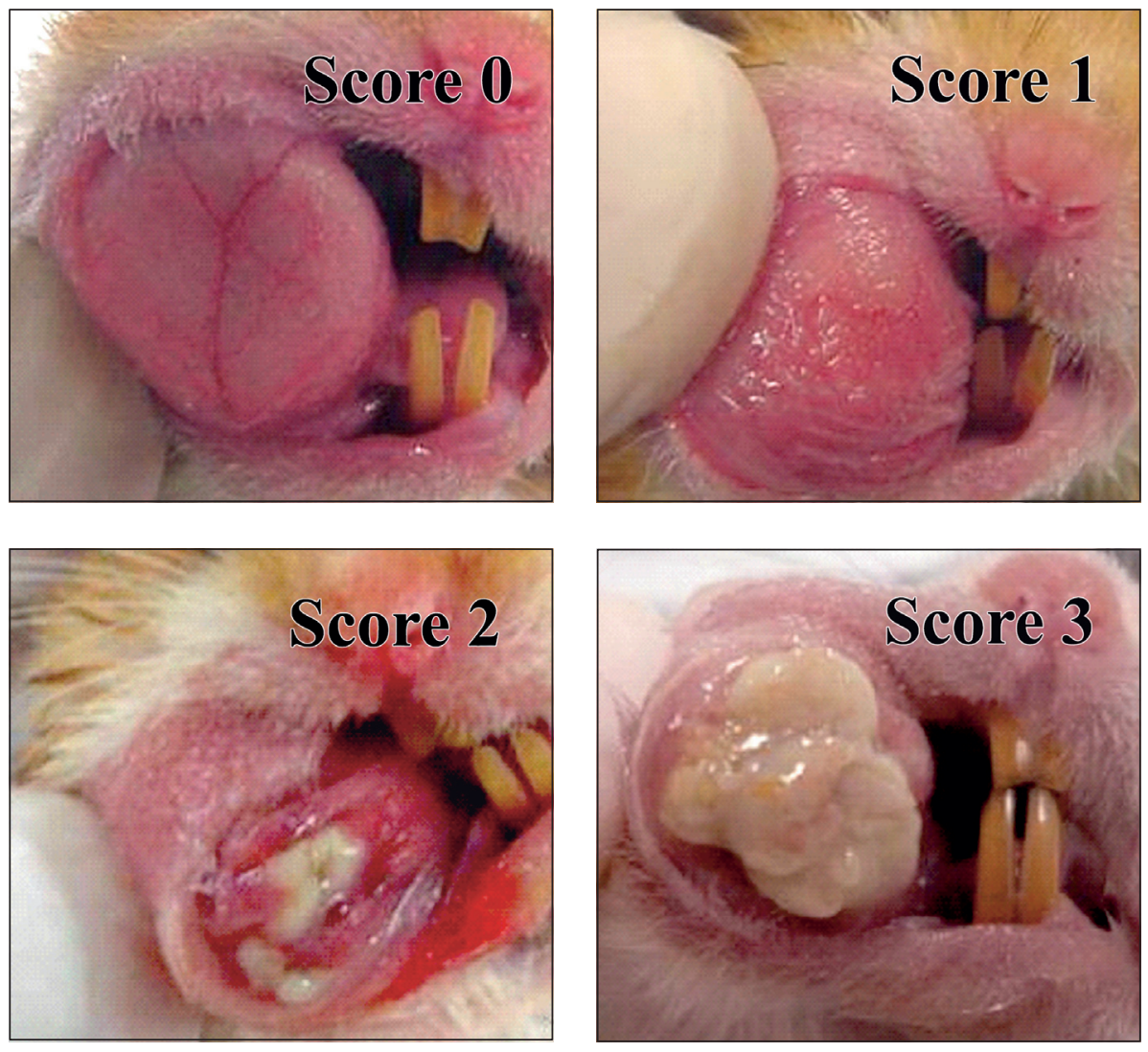

sa taken at the time of sacrifice were inserted in the PowerPoint program (Microsoft, Redmond, USA) for further classification using the scoring system proposed by Lima et al. ${ }^{11}$ as follows:

- Score 0 - normal pouch mucosa with no or mild erythema and hyperemia, no areas of bleeding, ulcerations or abscesses;

- Score 1 - moderate erythema and hyperemia, no hemorrhagic areas, ulcerations or abscesses;

- Score 2 - severe erythema and hyperemia, with hemorrhagic areas, small ulcers or blisters, but no abscesses;

- Score 3 - severe erythema and hyperemia, presence of hemorrhagic areas, extensive ulcerations and abscesses (Figure 2).

The rest of the animals were then sacrificed and the pouch mucosa was removed and fixed in $10 \%$ formalin for further histopathological analysis.

The fixed specimens were dehydrated and embedded in paraffin. Serial sections $5 \mu \mathrm{m}$ thick were obtained and stained with hematoxylin and eosin 
Table 1 - Mean \pm standard deviation of weight losses $(\mathrm{g})$ in different groups at different evaluation times (days).

\begin{tabular}{c|c|c|c|c|c}
\hline Experimental groups & 0 & 5 & 10 & 15 & $P$ values \\
\hline Control (G1) & $0.00 \pm 0.00^{\mathrm{A}, \mathrm{a}}$ & $26.00 \pm 8.71^{\mathrm{A}, \mathrm{a}}$ & $30.00 \pm 8.66^{\mathrm{A}, \mathrm{a}}$ & $18.33^{\mathrm{a}} \pm 13.33^{\mathrm{A}, \mathrm{a}}$ & 0.06 \\
\hline Preventive (G2) & $1.00 \pm 0.00^{\mathrm{A}, \mathrm{a}}$ & $27.00 \pm 3.74^{\mathrm{A}, \mathrm{a}}$ & $16.66 \pm 3.33^{\mathrm{A}, \mathrm{a}}$ & $21.00 \pm 11.55^{\mathrm{A}, \mathrm{a}}$ & 0.07 \\
\hline Therapeutic (G3) & $0.00 \pm 0.00^{\mathrm{A}, \mathrm{a}}$ & $16.00 \pm 2.44^{\mathrm{A}, \mathrm{b}}$ & $19.00 \pm 6.59^{\mathrm{A}, \mathrm{b}}$ & $3.33^{\mathrm{A}} \pm 1.66^{\mathrm{A}, \mathrm{a}, \mathrm{b}}$ & 0.006 \\
\hline Combined (G4) & $4.00 \pm 1.80^{\mathrm{A}, \mathrm{a}}$ & $13.00 \pm 2.54^{\mathrm{A}, \mathrm{a}}$ & $25.00 \pm 4.74^{\mathrm{A}, \mathrm{a}}$ & $21.66^{\mathrm{A}} \pm 16.66^{\mathrm{A}, \mathrm{a}}$ & 0.73 \\
\hline$P$ values & 0.31 & 0.16 & 0.52 & 0.59 & \\
\hline
\end{tabular}

Different uppercase letters indicate statistical differences between groups within the same evaluation times. Different lowercase lefters indicate statistical differences between evaluation times within the same group.

Table 2 - Mean \pm standard deviation of clinical scores of animals in different groups at different evaluation times (days).

\begin{tabular}{|c|c|c|c|c|c|}
\hline Experimental groups & 0 & 5 & 10 & 15 & $P$ values \\
\hline Control (G1) & $0.00 \pm 0.00^{A, a}$ & $1.00 \pm 0.00^{A, a, b}$ & $2.33 \pm 1.15^{\mathrm{A}, \mathrm{b}}$ & $1.00 \pm 1.00^{A, a, b}$ & $\geq 0.02$ \\
\hline Preventive (G2) & $0.00 \pm 0.00^{A, a}$ & $2.33 \pm 0.58^{\mathrm{B}, \mathrm{b}}$ & $2.00 \pm 1.00^{A, a, b}$ & $0.67 \pm 1.15^{A, a, b}$ & $\geq 0.03$ \\
\hline Therapeutic (G3) & $0.00 \pm 0.00^{A, a}$ & $0.33 \pm 0.58^{A, a}$ & $0.00 \pm 0.00^{B, a}$ & $2.00 \pm 1.73^{A, a}$ & $\geq 0.10$ \\
\hline Combined (G4) & $0.00 \pm 0.00^{A, a}$ & $0.00 \pm 0.00^{A, a}$ & $3.00 \pm 0.00^{A, b}$ & $0.67 \pm 1.15^{A, a}$ & $\geq 0.001$ \\
\hline$P$ values & 1.00 & $\geq 0.0005$ & $\geq 0.005$ & $\geq 0.60$ & \\
\hline
\end{tabular}

Different uppercase letters indicate statistical differences between groups within the same experimental times. Different lowercase letters indicate statistical differences between experimental times within the same group.

(H\&E). Each histological slide was classified using the scoring system proposed by Lima et al. ${ }^{11}$ as follows:

- Score 0 - normal epithelium and connective tissue without vasodilatation; absent or mild inflammatory infiltrate; absence of bleeding, ulcers and abscesses;

- Score 1 - mild vascular hyperemia; areas of reepithelialization; mild inflammatory infiltrate with a prevalence of mononuclear cells; no hemorrhagic areas, ulcerations or abscesses;

- Score 2 - moderate vascular redness; areas of epithelial degeneration; inflammatory infiltration with prevalence of neutrophils; hemorrhagic areas, edema and occasional ulcerations; absence of abscesses;

- Score 3 - severe hyperemia and vascular vasodilatation; inflammatory infiltration with prevalence of neutrophils; hemorrhagic areas, edema, extensive ulcerations and abscesses.

The ANOVA complemented by Tukey's test was used for multiple comparisons. The clinical and histological scores obtained by a blinded evaluator were adjusted in a completely randomized model for comparisons between times in relation to the set of groups and between groups in relation to the set of evaluation times. A factorial design model was adjusted for comparisons between groups and treatments with the set of times. The significance level was set at 5\%. All tests were performed using the SAS program for Windows, v.9.2 (SAS, Cary, USA).

\section{Results Weight analysis}

Weight losses ranging from 5\% to $10 \%$ occurred throughout the experiment. Weight losses were similar in the comparisons within the same experimental group at different evaluation times, except for G3 (Table 1). There were no significant differences in weight losses between the different experimental groups at the same evaluation times.

\section{Clinical analysis}

In Table 2, the comparisons between times within the same experimental groups show that the highest 
Table 3 - Mean \pm standard deviation of histopathological scores of animals in different groups at different evaluation times (days).

\begin{tabular}{c|c|c|c|c|c}
\hline Experimental groups & 0 & 5 & 10 & 15 & $P$ values \\
\hline Control $(G)$ & $0.00 \pm 0.00^{\mathrm{A}, \mathrm{a}}$ & $0.67 \pm 0.58^{\mathrm{A}, \mathrm{a}}$ & $2.00 \pm 1.73^{\mathrm{A}, \mathrm{a}}$ & $1.00^{\mathrm{a}} \pm 1.73^{\mathrm{A}, \mathrm{a}}$ & $\geq 0.28$ \\
\hline Preventive $(\mathrm{G} 2)$ & $0.00 \pm 0.00^{\mathrm{A}, \mathrm{a}}$ & $2.33 \pm 0.58^{\mathrm{B}, \mathrm{b}}$ & $2.67 \pm 0.58^{\mathrm{A}, \mathrm{b}}$ & $0.33^{\mathrm{A}} \pm 0.58^{\mathrm{A}, \mathrm{a}}$ & $\geq 0.002$ \\
\hline Therapeutic $(\mathrm{G} 3)$ & $0.00 \pm 0.00^{\mathrm{A}, \mathrm{a}}$ & $0.67 \pm 0.58^{\mathrm{A}, \mathrm{a}}$ & $0.00 \pm 0.00^{\mathrm{B}, \mathrm{a}}$ & $1.67^{\mathrm{A}} \pm 0.58^{\mathrm{A}, \mathrm{b}}$ & $\geq 0.004$ \\
\hline Combined (G4) & $0.00 \pm 0.00^{\mathrm{A}, \mathrm{a}}$ & $1.67 \pm 0.58^{\mathrm{A}, \mathrm{b}}$ & $3.00 \pm 0.00^{\mathrm{A}, \mathrm{c}}$ & $1.00 \pm 0.00^{\mathrm{A}, \mathrm{b}}$ & $\geq 0.0001$ \\
\hline$P$ values & 1.00 & $\geq 0.03$ & $\geq 0.01$ & $\geq 0.38$ & \\
\hline
\end{tabular}

Different uppercase letters indicate statistical differences between groups within the same experimental times. Different lowercase letters indicate statistical differences between experimental times within the same group.

clinical scores occurred at 10 days after 5-FU infusion, except in G3, in which the mean score remained at the baseline values throughout the experiment. In G1, mucositis followed a pattern that consisted of a significant increase in the score until Day 10, returning to the baseline value at the end of the experiment (Day 15). In G2, a significant increase in the score occurred on Day 5. In G3, the scores remained similar throughout the experimental period. In G4, there was a significant increase in the score through to Day 10, returning to the baseline values by Day 15 .

In the comparisons between groups within the same experimental times, there were no differences at the beginning (Day 0) and at the end (Day 15) of the experiment. G2 presented higher scores than the other groups on Day 5, and G3 showed lower scores than the other groups on Day 10. On Day 15, the mean score observed in G3 seemed higher than that of G1; however, statistical differences were not observed.

\section{Histological analysis}

In Table 3, the comparisons between times within the same group show that the highest scores occurred on Day 10 following 5-FU infusion, except in G3, in which the scores remained at the baseline values through to Day 10, with a significant increase on Day 15.

In the comparisons between groups within the same experimental times, there were no differences at the beginning and end of the experiment. G2 presented higher scores than all the other groups on Day 5. G3 presented the smallest scores on Day 10. On Day 15, the mean scores observed in this group were not statistically different, although they seemed higher than those of G1.

At the beginning and end of the experiment, no significant differences in histological scores were found among the groups.

\section{Comparison of clinical and histological scores}

Table 4 displays no significant differences among the groups, except for G4 on Day 5, in which the histopathological analysis resulted in significantly higher scores than those of the clinical analysis.

\section{Discussion}

LPT using the visible red spectrum has been found to reduce the severity of oral mucositis lesions as well as pain scores. ${ }^{3}$ For this reason, a laser emitting in the visible red $(660 \mathrm{~nm})$ range was used in the present study, with three different protocols to test possible preventive or therapeutic effects. This study investigated clinical features, including the animals' weight, performed a clinical analysis of the pouch mucosa in which mucositis was induced by 5-FU followed by local trauma, and also evaluated the histological aspects of the pouch mucosa in four experimental periods.

Weight loss can be an indicator of discomfort and pain while eating. ${ }^{10,13}$ Thus, the weight of the animals was determined at baseline and at time of sacrifice. Greater weight losses were expected in the Control group, which received no treatment. Surprisingly, the weight losses ranged from 5\% to $10 \%$ regardless of the experimental group. This may have occurred because the animals in the Control group had been spared from daily handling of their pouch 
Table 4 - Comparisons of the clinical and histopathological scores.

\begin{tabular}{|c|c|c|c|c|c|}
\hline \multirow{2}{*}{ Treatment } & \multirow{2}{*}{ Variable } & \multicolumn{4}{|c|}{ Time (days) } \\
\hline & & 0 & 5 & 10 & 15 \\
\hline Clinical & Control (G1) & $0.00 \pm 0.00^{\mathrm{A}}$ & $1.00 \pm 0.00^{\mathrm{A}}$ & $2.33 \pm 1.15^{\mathrm{A}}$ & $1.00 \pm 1.00^{\mathrm{A}}$ \\
\hline Histological & Control (G1) & $0.00 \pm 0.00^{\mathrm{A}}$ & $0.67 \pm 0.58^{A}$ & $2.00 \pm 1.73^{\mathrm{A}}$ & $1.00 \pm 1.73^{\mathrm{A}}$ \\
\hline \multicolumn{2}{|c|}{$P$ values } & 1.00 & 0.37 & 0.79 & 1.00 \\
\hline Clinical & Preventive (G2) & $0.00 \pm 0.00^{A}$ & $2.33 \pm 0.58^{A}$ & $2.00 \pm 1.00^{\mathrm{A}}$ & $0.67 \pm 1.15^{\mathrm{A}}$ \\
\hline Histological & Preventive (G2) & $0.00 \pm 0.00^{A}$ & $2.33 \pm 0.58^{A}$ & $2.67 \pm 0.58^{A}$ & $0.33 \pm 0.58^{A}$ \\
\hline \multicolumn{2}{|c|}{$P$ values } & 1.00 & 1.00 & 0.37 & 0.67 \\
\hline Clinical & Therapeutic (G3) & $0.00 \pm 0.00^{\mathrm{A}}$ & $0.33 \pm 0.58^{A}$ & $0.00 \pm 0.00^{A}$ & $2.00 \pm 1.73^{\mathrm{A}}$ \\
\hline Histological & Therapeutic (G3) & $0.00 \pm 0.00^{\mathrm{A}}$ & $0.67 \pm 0.58^{A}$ & $0.00 \pm 0.00^{A}$ & $1.67 \pm 0.58^{\mathrm{A}}$ \\
\hline \multicolumn{2}{|c|}{$P$ values } & 1.00 & 0.51 & 1.00 & 0.76 \\
\hline Clinical & Combined (G4) & $0.00 \pm 0.00^{\mathrm{A}}$ & $0.00 \pm 0.00^{\mathrm{A}}$ & $3.00 \pm 0.00^{A}$ & $0.67 \pm 1.15^{\mathrm{A}}$ \\
\hline Histological & Combined (G4) & $0.00 \pm 0.00^{\mathrm{A}}$ & $1.67 \pm 0.58^{B}$ & $3.00 \pm 0.00^{A}$ & $1.00 \pm 0.00^{\mathrm{A}}$ \\
\hline \multicolumn{2}{|c|}{$P$ values } & 1.00 & 0.007 & 1.00 & 0.64 \\
\hline
\end{tabular}

Different letters indicate statistical differences between clinical and histopathological scores within the same group at the same evaluation time.

mucosa, whereas the other animals, especially those receiving the preventive and combined treatments, were handled daily, probably provoking greater stress and discomfort.

The findings of the present study also demonstrate the positive effect of LPT in reducing the severity of mucositis when the therapeutic protocol was used. On Day 10, following 5-FU infusion, when an increase in the clinical and histological scores was expected, this group kept its initial scores, which were significantly lower than those of all the other groups. In other studies, this result was observed only on Day 15, when, in fact, it was already expected, because by this time, i.e., after the conclusion of chemotherapy, the oral mucositis would be self-resolved. ${ }^{14-15}$ Thus, our study revealed something new, i.e., it showed that the therapeutic protocol of LPT presented a positive effect exactly when the clinical signs of oral mucositis are more critical.

It is difficult to understand why the therapeutic protocol group presented a positive response to the LPT, whereas the preventive protocol group and even the combined protocol group had no effect at all. It is known that LPT has effects only on stressed or diseased tissues. This therapy is able to restore the regular metabolic potential of stressed cells. ${ }^{16-17}$

With this in mind, it could be posited that the initial irradiation on an already stressed tissue-as occurred in the therapeutic protocol group-may have led to a prompt response to the LPT, whereas the tissue that started to be irradiated when it was in a regular metabolic condition could have been inhibited using a relatively high dose of daily irradiation. Concurring with this hypothesis, França $e t$ al. ${ }^{13}$ have observed positive results in preventive protocols by using LPT in smaller dose. On the other hand, the absence of positive results of LPT, when applied in the preventive and combined protocols, may be explained by the fact that the animals of this group were handled more often than those of the therapeutic protocol group. This probably excessive manipulation could have caused greater stress, which may also explain the weight losses observed in the animals of these groups. The manipulation of these animals started five days before the infusion, with the hypothesis that this early LPT application could have a protective effect on the mucosa. It is possible that different results could have been obtained for the present study if the preventive and the combined protocols had started on the infusion day, as previously described by França et al. ${ }^{13}$

Further in vivo studies involving animal models should be conducted to determine the beneficial effect of LPT in controlling oral mucositis, and also to 
obtain a better understanding of this process. This could lead to clinical protocols for the prevention of mucositis, which is the main goal of health professionals who deal with this common complication of cancer treatment that may often be decisive in regard to patient survival.

\section{Conclusion}

This study showed that the positive effects of oral mucositis management were obtained only when the

\section{References}

1. Sonis ST. Pathobiology of mucositis. Semin Oncol Nurs. 2004 Feb;20(1):11-5.

2. Elting LS, Cooksley C, Chambers M, Cantor SB, Manzullo E, Rubenstein EB. The burdens of cancer therapy. Clinical and economic outcomes of chemotherapy-induced mucositis. Cancer. 2003 Oct;98(7):1531-9.

3. Schubert MM, Eduardo FP, Guthrie KA, Franquin JC, Bensadoun RJ, Migliorati CA, et al. A phase III randomized doubleblind placebo-controlled clinical trial to determine the efficacy of low level laser therapy for the prevention of oral mucositis in patients undergoing hematopoietic cell transplantation. Support Care Cancer. 2007 Oct;15(10):1145-54.

4. Eduardo FP, Bezinelli L, Luiz AC, Correa L, Vogel C, Eduardo CP. Severity of oral mucositis in patients undergoing hematopoietic cell transplantation and an oral laser phototherapy protocol: a survey of 30 patients. Photomed Laser Surg. 2009 Feb;27(1):137-44.

5. Peterson DE, Bensadoun RJ, Roila F. Management of oral and gastrointestinal mucositis: ESMO Clinical Practice Guidelines. Ann Oncol. 2011 Sep;22 Suppl 6:vi78-84.

6. Bjordal JM, Bensadoun RJ, Tunèr J, Frigo L, Gjerde K, Lopes-Martins RA. A systematic review with meta-analysis of the effect of low-level laser therapy (LLLT) in cancer therapy-induced oral mucositis. Support Care Cancer. 2011 Aug;19(8):1069-77.

7. Bensadoun RJ, Franquin JC, Ciais G, Darcourt V, Schubert $\mathrm{MM}$, Viot M, et al. Low-energy He/Ne laser in the prevention of radiation-induced mucositis. A multicenter phase III randomized study in patients with head and neck cancer. Support Care Cancer. 1999 Jul;7(4):244-52.

8. Simões A, Eduardo FP, Luiz AC, Campos L, Sá PH, Cristófaro $\mathrm{M}$, et al. Laser phototherapy as topical prophylaxis against head and neck cancer radiotherapy-induced oral mucositis: comparison between low and high/low power lasers. Lasers Surg Med. 2009 Apr;41(4):264-70.

9. Sonis ST, Tracey C, Shklar G, Jenson J, Florine D. An animal model for mucositis induced by cancer chemotherapy. Oral Surg Oral Med Oral Pathol. 1990 Apr;69(4):437-43.
LPT was applied in the therapeutic protocol (from $\mathrm{D}+5$ to $\mathrm{D}+15$ after chemotherapy).

\section{Acknowledgments}

The authors would like to thank the state governmental foundation, "Fundação de Amparo à Pesquisa do Estado de São Paulo (FAPESP)," for funding this research (Grant \#2009/10254-4), Mr. Richard Boike and Ms. Nancy Cristina Martorana for the revision of the English language.

10. Sonis ST, Van Vugt AG, Brien JP, Muska AD, Bruskin AM, Rose A, et al. Transforming growth factor-beta 3 mediated modulation of cell cycling and attenuation of 5-fluorouracil induced oral mucositis. Oral Oncol. 1997 Jan;33(1):47-54.

11. Lima V, Brito GA, Cunha FQ, Rebouças CG, Falcão BA, Augusto RF, et al. Effects of the tumour necrosis factor-alpha inhibitors pentoxifylline and thalidomide in short-term experimental oral mucositis in hamsters. Eur J Oral Sci. 2005 Jun;113(3):210-7.

12. Leitão RF, Ribeiro RA, Bellaguarda EA, Macedo FD, Silva LR, Oriá RB, et al. Role of nitric oxide on pathogenesis of 5 -fluorouracil induced experimental oral mucositis in hamster. Cancer Chemother Pharmacol. 2007 Apr;59(5):603-12.

13. França CM, França CM, Núñez SC, Prates RA, Noborikawa E, Faria MR, Ribeiro MS et al. Low-intensity red laser on the prevention and treatment of induced-oral mucositis in hamsters. J Photochem Photobiol B. 2009 Jan;94(1):25-31.

14. Lopes NN, Plapler H, Chavantes MC, Lalla RV, Yoshimura EM, Alves MT. Cyclooxygenase- 2 and vascular endothelial growth factor expression in 5-fluorouracil-induced oral mucositis in hamsters: evaluation of two low-intensity laser protocols. Support Care Cancer. 2009 Nov;17(11):1409-15.

15. Lopes NN, Plapler H, Lalla RV, Chavantes MC, Yoshimura EM, Silva MA, et al. Effects of low-level laser therapy on collagen expression and neutrophil infiltrate in 5-fluorouracilinduced oral mucositis in hamsters. Lasers Surg Med. 2010 Aug;42(6):546-52.

16. Pereira AN, Eduardo CP, Matson E, Marques MM. Effect of low-power laser irradiation on cell growth and procollagen synthesis of cultured fibroblasts. Lasers Surg Med. 2002 Oct;31(4):263-7.

17. Marques MM, Pereira AN, Fujihara NA, Nogueira FN, Eduardo CP. Effect of low-power laser irradiation on protein synthesis and ultrastructure of human gingival fibroblasts. Lasers Surg Med. 2004 Mar;34(3):260-5. 